Energy Materials and Solar Cells

Elsevier Editorial System(tm) for Solar

Manuscript Draft

Manuscript Number: SOLMAT-D-18-01491R1

Title: Side-pumped continuous-wave Nd:YAG solar laser with 5.4\% slope efficiency

Article Type: Full Length Article

Keywords: Solar-pumped laser; slope efficiency; Nd:YAG; lower-order mode; doughnut-shaped; TEMO0-mode.

Corresponding Author: Dr. Dawei Liang, Ph.D.

Corresponding Author's Institution: Universidade Nova de Lisboa

First Author: Dawei Liang, Ph.D.

Order of Authors: Dawei Liang, Ph.D.; Cláudia R Vistas, PhD; Joana Almeida, PhD; Bruno D Tibúrcio, MSc; Dário Gracia, MSc

Abstract: We report here a significant progress in side-pumped solar laser slope efficiency by pumping a $3.0 \mathrm{~mm}$ diameter, $30 \mathrm{~mm}$ length $\mathrm{Nd}$ :YAG single-crystal laser rod through a heliostat-parabolic mirror solar energy collection and concentration system with $0.9 \mathrm{~m} 2$ effective collection area. A large fused silica aspheric lens allowed an efficient focusing of the concentrated solar power from the focal zone of the parabolic mirror into the laser rod mounted within a novel twodimensional semi-cylindrical pump cavity. 15.3 W continuous-wave $1064 \mathrm{~nm}$ solar laser power was measured, resulting in 5.40\% slope efficiency and 2.43\% solar-to-laser conversion efficency, being 2.08 and 2.30 times, respectively, more than the previous records by side-pumping configuration. The 5.40\% slope efficiency is also 1.08 times more than the previous record with Nd:YAG single-crystal rod by end-side-pumping approach. By adopting an asymmetric laser resonator, this novel sidepumping scheme also enabled an efficient production of high-quality solar laser beams in either TEM11 (4.0 W), TEM01* (doughnut-shaped, 2.9 W), TEM10 (3.2 W) or TEMOO (2.8 W) mode profiles.

Research Data Related to this Submission

There are no linked research data sets for this submission. The following reason is given:

Data will be made available on request 


\section{Dear Reviewer \#1:}

Many thanks for your insightful and instructive reviews.

I would like to answer your comments one by one:

1. For different solar irradiance led to different thermal lensing effect, different TEM00 mode laser power at different solar power level. As described in the manuscript, the larger the pumping power with the smaller the laser power, varying between $770 \mathrm{~W} / \mathrm{m} 2$ and $830 \mathrm{~W} / \mathrm{m} 2$. Is there any solution to solve the thermal lens problem?

Yes. Many thanks

I have added the following text to a new "Discussions" section in the revised manuscript to answer the concerns and instructions of both reviewers

"During the experiment, we have noticed a very interesting phenomenon that the solar laser slope efficiency actually reached the maximum value of $5.4 \%$ at $700 \mathrm{~W} / \mathrm{m}^{2}$ solar irradiance when the Sun reached us through a homogeneous, thin layer of cloud. Only $5.1 \%$ slope efficiency was measured at $770 \mathrm{~W} / \mathrm{m}^{2}$ irradiance through a more transparent sky condition. Before reaching the laser rod, a considerable part of IR solar radiation from the Sun was already absorbed by the cloud, three conclusions may therefore be drawn. 1: Our solar laser can offer higher slope efficiency with the thin layer of could in the sky than without it. 2: More importantly, for cloudless sunny days, an IR filter may be directly integrated to the solar laser head so that only less than $0.9 \mu \mathrm{m}$ wavelength solar radiation is used to pump the rod. 3: It is hence not difficult to infer that another UV filter may also be added to cut undesirable solar radiation below $0.45 \mu \mathrm{m}$. The above mentioned measures may consequently ensure a slope efficiency higher than $5.4 \%$ in future researches.

In solid-state lasers, end-side-pumped scheme usually produces higher laser system efficiency as compared to that by side-pumping approach. Indeed, the end-side-pumped Nd:YAG [13] solar laser enabled $3.1 \%$ solar-to-laser power conversion efficiency, being 1.24 times higher than that of our side-pumped Nd:YAG laser, as given in Fig.6. However....."

2. The solar laser beam quality M2 factors were measured in Section 4, however, it would be better if the beam quality M2 factors of lower order mode solar laser be increased.

As reviewer 1 have noticed, from Table 2 (now Table 1), a few-mode and TEM $\mathrm{O}_{00}$-mode laser beam profiles were strongly dependent on the thermal lensing effect of the laser rod. A slight change in solar irradiance during the experiment or the shift of the solar pump focal spot due to the tracking error of the heliostat will result in the final alteration of laser rod thermal focal length, which makes classical $\mathrm{M}^{2}$ factor measurement much more difficult than that for multimode laser beam. Luckily, a few-mode and $\mathrm{TEM}_{\mathrm{Oo}}$-mode laser beam profiles (and consequently their associated $\mathrm{M}^{2}$ factors) were clearly monitored by CINOGY laser beam profiler as shown in Table 2 ( now Table 1), indicating a relatively strong influence of solar irradiance on these beam profiles. 


\section{Dear Reviewer \#2:}

Many thanks for your insightful reviews and instructions on how to improve our manuscript I would like to answer your comments one by one:

1) Line 14 --change we as "The authors"

Yes, we have changed "We" as "The authors"

2) Too much explanation of the content quoted in the references making the introduction part voluminous. Instead the limitations of this w.r.t end side pumping would have been discussed.

Yes, the following text was added to the "Discussions" section

"In solid-state lasers, end-side-pumped scheme usually produces higher laser system efficiency as compared to that by side-pumping approach. Indeed, the end-side-pumped Nd:YAG [13] solar laser enabled 3.1\% solarto-laser power conversion efficiency, being 1.24 times higher than our side-pumped Nd:YAG laser as given in Fig.6. However......"

3) In Lines 48 to 60 the content in the references is explained and the same is presented again in a tabular form in Table-1.Duplication in text form and tabular form is not necessary

Yes, Many thanks

Both Table 1 and its corresponding text, from page 2 line 52 to page 3 line 2, were omitted in the revised manuscript

4) Main focus in the paper is on improvement of slope efficiency and conversion efficiency, which could have been shown in Fig-6 comparatively highlighting the same.

Yes, many thanks for your instructions.

To highlight the slope efficiency and also to answer the concerns of Reviewer 1, I have added the following text to a new "Discussion" section in the revised manuscript.

"During the experiment, we have noticed a very interesting phenomenon that the solar laser slope efficiency actually reached the maximum value of $5.4 \%$ at $700 \mathrm{~W} / \mathrm{m}^{2}$ solar irradiance when the Sun reached us through a homogeneous, thin layer of cloud. Only $5.1 \%$ slope efficiency was measured at $770 \mathrm{~W} / \mathrm{m}^{2}$ irradiance 
through a more transparent sky condition. Before reaching the laser rod, a considerable part of IR solar radiation from the Sun was already absorbed by the cloud, three conclusions may therefore be drawn. 1: Our solar laser can offer higher slope efficiency with the thin layer of could in the sky than without it. 2: More importantly, for cloudless sunny days, an IR filter may be directly integrated to the solar laser head so that only less than $0.9 \mu \mathrm{m}$ wavelength solar radiation is used to pump the rod. 3 : It is hence not difficult to infer that another UV filter may also be added to cut undesirable solar radiation below $0.45 \mu \mathrm{m}$. The above mentioned measures may consequently ensure a slope efficiency higher than $5.4 \%$ in future researches.

In solid-state lasers, end-side-pumped scheme usually produces higher laser system efficiency as compared to that by side-pumping approach. Indeed, the end-side-pumped Nd:YAG [13] solar laser enabled $3.1 \%$ solar-to-laser power conversion efficiency, being 1.24 times higher than our side-pumped Nd:YAG laser as given in Fig.6. However...."

5) In the conclusion part discussion on reference papers may be omitted to bring more clarity. In page 11 lines 12 to 30 can be shifted to discussion part/results in the paper.

Yes, many thanks

Page 11 lines 12-30 were shifted to a new "Discussions" section in order to bring more clarity.

\section{Discussions on reference papers were also transferred from "Conclusions" section to "Discussions" section}




\section{Highlights}

1. $5.40 \%$ slope efficiency was achieved, being 2.08 times more than the previous side-pumping record.

2. $2.43 \%$ solar-to-laser conversion efficiency was attained, being 2.30 times more than the previous side-pumping record.

3. An efficient production of high-quality solar laser beams with either $\mathrm{TEM}_{11}(4.0 \mathrm{~W}), \mathrm{TEM}_{01 *}$ (doughnut-shaped, $\left.2.9 \mathrm{~W}\right), \mathrm{TEM}_{10}(3.2 \mathrm{~W})$ or $\operatorname{TEM}_{00}(2.8 \mathrm{~W})$ mode profiles. 


\title{
Side-pumped continuous-wave Nd:YAG solar laser with $5.4 \%$ slope efficiency
}

\author{
Dawei Liang", Cláudia R. Vistas, Joana Almeida, Bruno D. Tibúrcio and Dário Garcia \\ CEFITEC, Departamento de Física, FCT, Universidade NOVA de Lisboa, 2829-516, Campus de Caparica, Portugal \\ *d1@fct.unl.pt
}

\begin{abstract}
The authors report here a significant progress in side-pumped solar laser slope efficiency by pumping a $3.0 \mathrm{~mm}$ diameter, $30 \mathrm{~mm}$ length Nd:YAG single-crystal laser rod through a heliostat-parabolic mirror solar energy collection and concentration system with $0.9 \mathrm{~m}^{2}$ effective collection area. A large fused silica aspheric lens allowed an efficient focusing of the concentrated solar power from the focal zone of the parabolic mirror into the laser rod mounted within a novel two-dimensional semicylindrical pump cavity. $15.3 \mathrm{~W}$ continuous-wave $1064 \mathrm{~nm}$ solar laser power was measured, resulting in $5.40 \%$ slope efficiency and $2.43 \%$ solar-to-laser conversion efficency, being 2.08 and 2.30 times, respectively, more than the previous records by side-pumping configuration. The $5.40 \%$ slope efficiency is also 1.08 times more than the previous record with Nd:YAG single-crystal rod by end-side-pumping approach. By adopting an asymmetric laser resonator, this novel side-pumping scheme also enabled an efficient production of high-quality solar laser beams in either $\mathrm{TEM}_{11}(4.0$ $\mathrm{W}), \mathrm{TEM}_{01}{ }^{*}$ (doughnut-shaped, $\left.2.9 \mathrm{~W}\right), \mathrm{TEM}_{10}(3.2 \mathrm{~W})$ or $\mathrm{TEM}_{00}(2.8 \mathrm{~W})$ mode profiles.
\end{abstract}

Keywords: Solar-pumped laser; slope efficiency; Nd:YAG; lower-order mode; doughnutshaped; $\mathrm{TEM}_{00}$-mode.

\section{Introduction}

Solar-pumped laser can be considered as one of the most promising technologies for both renewable energy and laser technology researches. As solar energy is highly abundant and most importantly, free, the direct production of solar laser power by natural sunlight may provide cost-effective solutions to many demanding applications of laser technologies. Since no artificial pumping sources like expensive laser diode stacks or arc lamps along with their associated electrical power generation and conditioning equipment are involved, solar laser are more adequate for space applications, such as atmospheric and ocean sensing, laser power beaming, deep space communications [1], etc. Solar laser has also large potentials for terrestrial applications like high-temperature material processing and magnesium-hydrogen energy cycle $[2,3]$.

Young [4] and other researchers [5-9] were early pioneers for successful continuous-wave (cw) $1064 \mathrm{~nm}$ Nd:YAG solar laser emissions, from $1.0 \mathrm{~W}$ [4] in 1966 to $60.0 \mathrm{~W}$ in 1988 [6], pumped through a primary parabolic mirror, along with a secondary and a tertiary Compound Parabolic Concentrators (CPC) [9]. Solar laser collection efficiency was successfully boosted to $6.7 \mathrm{~W} / \mathrm{m}^{2}$ in 2003 [9]. Most significant progresses in solar laser collection efficiency have been made in the last decade with end-side-pumping approaches $[10-15] .18 .7 \mathrm{~W} / \mathrm{m}^{2}$ solar laser collection efficiency was reported by end-side-pumping a large Cr:Nd:YAG ceramic laser rod through a $1.3 \mathrm{~m}^{2}$ area Fresnel lens in 2007 [10]. $19.3 \mathrm{~W} / \mathrm{m}^{2}$ laser collection efficiency was later achieved by also end-side-pumping a small Nd:YAG single-crystal rod through a $0.64 \mathrm{~m}^{2}$ area Fresnel lens in 2011 [11]. $30.0 \mathrm{~W} / \mathrm{m}^{2}$ laser collection efficiency was attained by end-side-pumping a large Nd:YAG rod through a $4.0 \mathrm{~m}^{2}$ area Fresnel lens in 2012 [12]. 
By end-side-pumping a $4.0 \mathrm{~mm}$ diameter, $35 \mathrm{~mm}$ length $\mathrm{Nd}$ : $\mathrm{YAG}$ single-crystal rod through an heliostatparabolic mirror solar energy concentration system with $1.18 \mathrm{~m}^{2}$ effective collection area, $37.2 \mathrm{~W} \mathrm{cw}$ multimode solar laser power was achieved in 2017 , reaching $31.5 \mathrm{~W} / \mathrm{m}^{2}$ collection efficiency and the highest laser slope efficiency of $5.25 \%$ [13]. Also in end-side-pumping configuration, $32.1 \mathrm{~W} / \mathrm{m}^{2}$ collection efficiency and 5.0\% slope efficiency were obtained by pumping a large composite Nd:YAG rod through a $1.03 \mathrm{~m}^{2}$ area Fresnel lens in 2018 [14]. Most recently, a monolithic fused silica liquid light guide lens was used to achieve end-side-pumping of a $4.5 \mathrm{~mm}$ diameter $35 \mathrm{~mm}$ length Cr:Nd:YAG ceramic laser rod within a conical pumping cavity. $32.5 \mathrm{~W} / \mathrm{m}^{2}$ solar laser collection efficiency and the state-of-the-art laser slope efficiency of $6.7 \%$ were achieved through a heliostat-parabolic mirror system [15].

Although today's most efficient solar laser systems are all in end-side-pumping configurations [10-15], side-pumping approach is also a very attractive choice due to the following reasons:

1: The solar laser beam brightness from a side-pumping configuration [16-19] is usually much higher than those by end-side-pumping configuration [10-15]. Side-pumping configuration allows free access to both rod ends and permits the optimization of more laser resonator parameters, improving largely the laser beam quality and enabling the efficient extraction of solar laser power in low-order, fundamental or even doughnut-shaped mode profile. For many laser applications, it is highly desirable to operate a laser in the lowest-mode possible because of its low divergence and ability to be focused into a doughnutshaped or a diffraction-limited light spot. Side-pumping is also very adequate for laser power scaling as it gives uniform absorption along the rod axis and spreads the absorbed power within the laser medium, reducing hence the associated thermal loading problems.

2: Another advantage of side-pumped solar laser lies in its excellent solar tracking error compensation capacity as compared to that of the end-side-pumped configuration. In side-pumping configuration, solar tracking error in azimuth axis only influences the shift of focal spot along the laser rod, usually mounted in azimuth direction. The absorbed solar pump power within a pump cavity is therefore not considerably affected by the tracking errors in azimuth axis, as long as the focused spot still stays within the pump cavity. Also since the variation of solar tracking error in altitude axis is not significant, especially at noon, we can usually achieve more stable solar laser output by side-pumping configuration. For end-sidepumping, however, an important part of the concentrated solar power at the focus is directly focused onto the end face of a laser rod, any slight solar tracking error in either azimuth or altitude axis can cause a significant reduction or even extinction in laser output power, hampering hence its stable emission.

Indeed, all the previous researches from 1966 to 2003 were in side-pumping configuration [4-9]. Side-pumped solar laser history started from the first solar-pumped laser $(1 \mathrm{~W}$, at $0.36 \%$ solar-to-laser conversion efficiency) by Young in 1966 [4], followed by the gradual improvement in solar laser output power by both Arashi (18 W) in 1984 [5] and Weksler \& Shwartz (60 W, at 1.17\% slope efficiency) in 1988 [6]. Solar laser collection efficiency was improved to $4.7 \mathrm{~W} / \mathrm{m}^{2}$ and solar-to-laser conversion efficiency also improved to $0.495 \%$ by Jenkins et. al. in 1996 [8]. $6.7 \mathrm{~W} / \mathrm{m}^{2}$ collection efficiency was achieved by Lando et. al. [9] in 2003, but its solar-to-laser conversion efficiency was limited to only 0.71\%. In recent years, Almeida et. al. in 2012 [16], Liang et.al. in 2013 [17, 18], Vistas et. al. in 2016 [19] gradually improved side-pumped laser collection efficiency to about $12.0 \mathrm{~W} / \mathrm{m}^{2}$, solar-to-laser conversion efficiency to nearly $1.0 \%$ and laser slope efficiency to between $2.2 \%-3.1 \%$.

A large advance in side-pumped solar laser slope efficiency and solar-to-laser conversion efficiency is introduced here. The aspheric fused silica lens allowed an efficient focusing of concentrated solar pump power from the focal zone of the parabolic mirror into the thin laser rod mounted within the twodimensional semi-cylindrical pump cavity. $15.3 \mathrm{~W} \mathrm{cw} 1064 \mathrm{~nm}$ solar laser power was measured, corresponding to $17.0 \mathrm{~W} / \mathrm{m}^{2}$ collection efficiency, $5.40 \%$ slope efficiency and $2.43 \%$ solar-to-laser power conversion efficency, being $1.45,2.3$, and 2.15 times, respectively, more than the previous records in side-pumping configuration [17]. The measured system slope efficiency is already 1.08 times higher than end-side-pumped solar laser with Nd:YAG single-crystal rod [13]. It is only $19.4 \%$ less than that of end-side-pumped Cr:Nd:YAG solar laser [15]. Besides, our novel side-pumping approach enabled an 
efficient production of high-quality solar laser beams with very useful $\mathrm{TEM}_{11}, \mathrm{TEM}_{01 *}, \mathrm{TEM}_{10}, \mathrm{TEM}_{00}$ mode profiles.

\section{NOVA Heliostat-Parabolic Mirror Solar Laser System}

\subsection{Solar energy collection and concentration system of NOVA University}

NOVA heliostat-parabolic mirror solar laser system in Fig. 1 was composed of a large plane mirror (composed of 4 flat segments) with $93.5 \%$ reflectivity, mounted on a two-axis heliostat, which redirected the incoming solar radiation towards a stationary parabolic mirror with $1.5 \mathrm{~m}$ diameter, $60^{\circ}$ rim angle and $660 \mathrm{~mm}$ focal length.

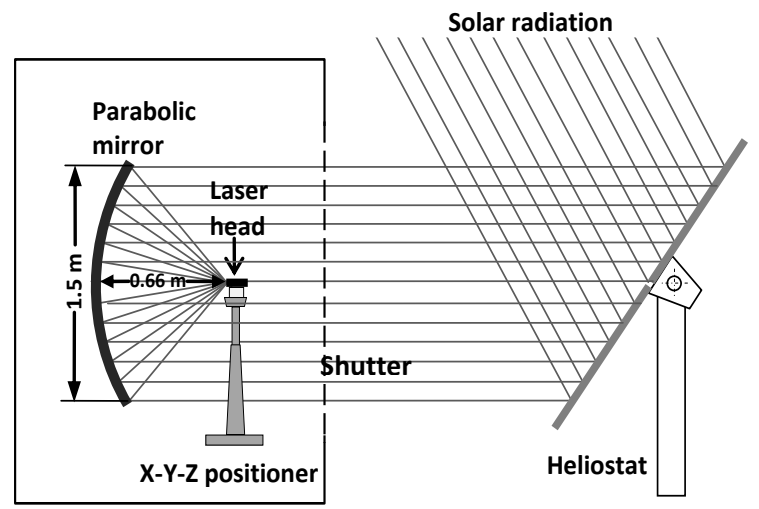

Fig. 1 Schematics of NOVA stationary heliostat-parabolic mirror solar energy collection and concentration system.

The parabolic mirror was back-surface silver coated. Because of iron impurities within $10 \mathrm{~mm}$ glass substrates, $80.0 \%$ reflectivity was measured for this primary mirror. The total combined reflectance of both the heliostat and the parabolic mirror was $74.8 \%$. To avoid overheating the ceramic active medium, we limited the input solar power at focus by masking the external annular area of the $1.5 \mathrm{~m}$ diameter mirror, so that only its $1.16 \mathrm{~m}$ diameter central circular area was utilized. $0.9 \mathrm{~m}^{2}$ effective solar collection area was calculated by discounting the shadowing area of $0.15 \mathrm{~m}^{2}$ by both the laser head, its supporting mechanics, and the X-Y-Z positioner. For $700 \mathrm{~W} / \mathrm{m}^{2}$ solar irradiance in August 2018 in Lisbon area, about $470 \mathrm{~W}$ solar power was measured in the focal zone.

2.2 Solar laser head with the large fused silica aspheric lens, the Nd:YAG rod and the two-dimensional semicylindrical pump cavity

As shown in Fig. 2, the solar laser head was composed of the fused silica aspheric lens and the 2D semicylindrical pump cavity within which the $3.0 \mathrm{~mm}$ diameter, $30 \mathrm{~mm}$ length Nd:YAG single-crystal rod was efficiently pumped. The $80 \mathrm{~mm}$ diameter fused silica aspheric lens had an aspherical input face with $44 \mathrm{~mm}$ radius of curvature, -0.002 conic factor and a plane output face, coupling efficiently the concentrated solar radiation from the focal zone into the $3.0 \mathrm{~mm}$ diameter laser rod through cooling water. There was $6 \mathrm{~mm}$ space between this plane output face and the laser rod, as shown in Fig. 2 and Fig.3. The maximum contact between the coolant and the rod was essential for the removal of the generated heat. Both fused silica material and cooling water were also useful for partially preventing both UV solarization and IR heating to the laser rod. The water cooling scheme is indicated by Fig.2. Arrows indicate water flow directions, passing firstly below the pump cavity and cool the rod from two sides and finally exit the pump cavity through the spaces between the aspheric lens and the laser rod. Both the aspheric lens and the pump cavity were all actively cooled by water at $6 \mathrm{~L} / \mathrm{min}$ flow rate. The inner wall of the two-dimensional semicylindrical pumping cavity was bonded with a protected silver-coated aluminum foil with $94 \%$ reflectivity. The solar laser head was fixed on an X-Y-Z axes positioner, ensuring an accurate optical alignment of the laser head within the focal zone, as shown by the right inset photo of Fig. 2. One part of the concentrated radiation was directly focused onto the upper surface of the $3.0 \mathrm{~mm}$ diameter, $30 \mathrm{~mm}$ length $\mathrm{Nd}$ :YAG rod by the fused silica aspheric lens itself. Double-pass pumping to the $3.0 \mathrm{~mm}$ diameter laser rod was also possible in this case by the semi-cylindrical pump cavity, as demonstrated by Fig. 3. A significant part of pump rays exiting the aspheric lens did not pass directly through the laser rod, hence we can understand great usefulness of the two-dimensional semi-cylindrical pump cavity 
with $\mathrm{R}=4.5 \mathrm{~mm}$ radius of curvature, $\mathrm{W}=9.0 \mathrm{~mm}$ width and $\mathrm{H}=7.0 \mathrm{~mm}$ depth. With this novel pump cavity, pump rays from the aspheric lens were both reflected and focused sharply into the $3.0 \mathrm{~mm}$ diameter laser rod, as shown in Fig. 3, ensuring the final enhancements in both solar laser efficiency and beam quality, as compared to other sidepumping schemes $[16-19]$.

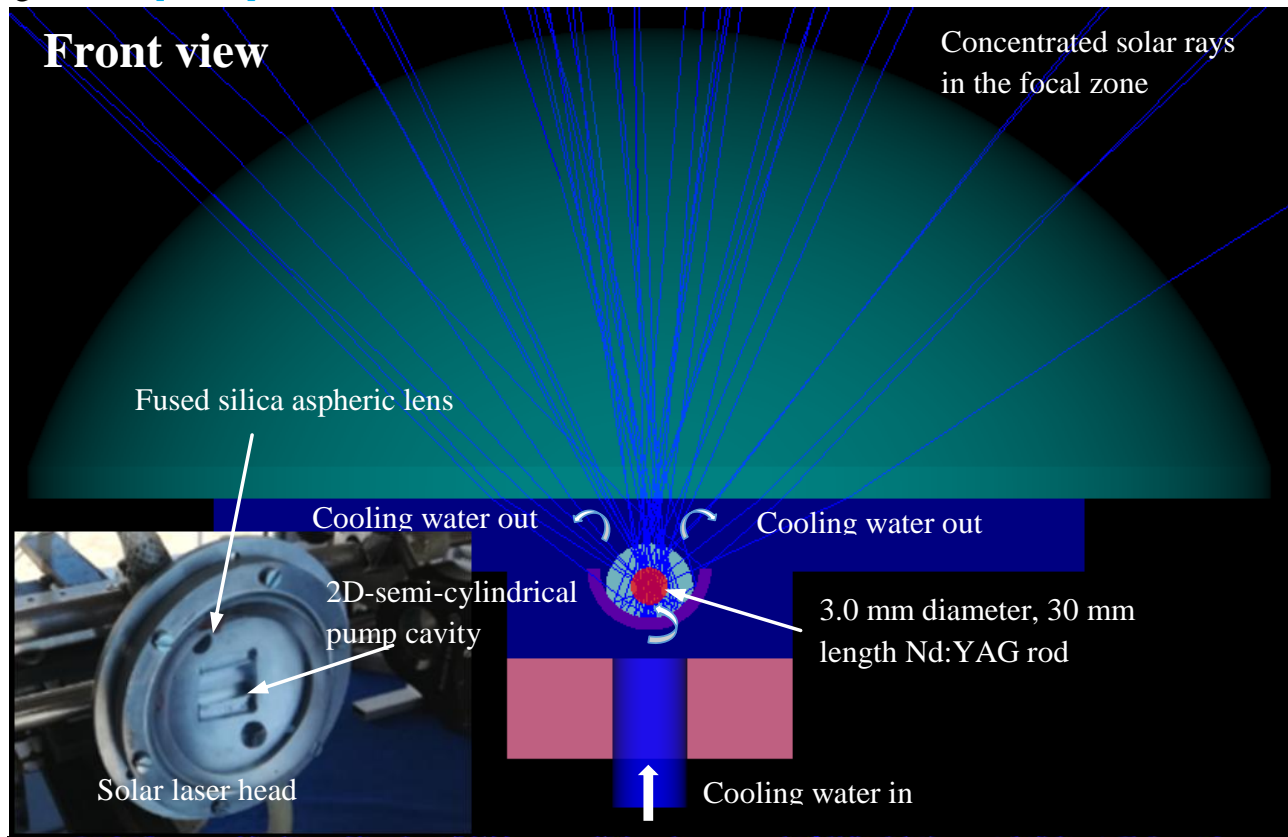

Side-view

Fused silica aspheric lens
Concentrated solar rays in the focal zone 

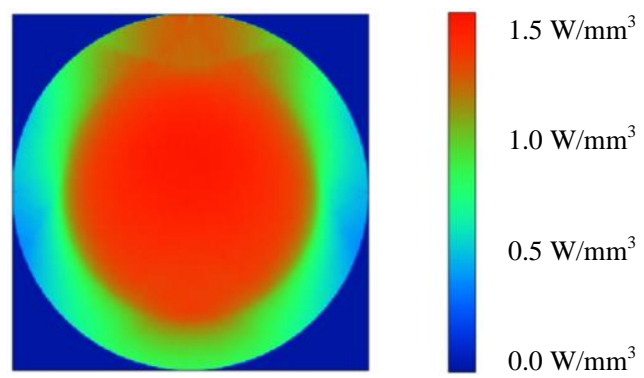

Fig. 4. Absorbed pump-flux distributions along central transversal cross-section of the $3.0 \mathrm{~mm}$ diameter, $30 \mathrm{~mm}$ length Nd:YAG single-crystal rod

Absorbed pump light distributions presented a circularly symmetric near-uniform distribution along the laser rod, red color means near maximum pump absorption, whereas blue means little or no absorption. When the absorption profile was centrally peaked, the temperature on the rod axis increased further, resulting in stronger thermal lensing at the center, higher-order aberrations at the periphery, and larger stress in the laser rod. Consequently, it was very important for us to achieve the circularly symmetric absorbed pump flux distribution in the laser rod by optimizing carefully both the parameters of fused silica aspheric lens and the two-dimensional semi-cylindrical cavity. The absorbed pump flux data from the $\mathrm{ZEMAX}^{\odot}$ analysis was then processed by LASCAD $^{\odot}$ software to optimize solar laser output performances.

Laser cavity analysis and design $\left(\mathrm{LASCAD}^{\circledR}\right)$ codes were then used to optimize laser output power and beam quality. The effective pump power of the light source took into account about $16 \%$ overlap between the absorption spectrum of the 1.0 at.\% Nd:YAG medium and the solar spectrum [21]. The stimulated emission cross-section of $2.8 \times 10^{-19} \mathrm{~cm}^{2}$, the fluorescence life time of $230 \mu$ s and a typical absorption and scattering loss of $0.003 \mathrm{~cm}^{-1}$ for the laser medium were adopted in LASCAD ${ }^{\odot}$ analysis. The mean absorbed and intensity-weighted solar pump wavelength of $660 \mathrm{~nm}$ [6] was also used in the analysis. $L_{R}$ represents the laser rod length, $L_{1}$ represents the separation between HR1064nm mirror and 
left AR1064 nm end face of the laser rod and $\mathrm{L}_{2}$ represents the separation between the right AR1064nm end face and PR 1064 nm output mirror, as shown in Fig. 5.

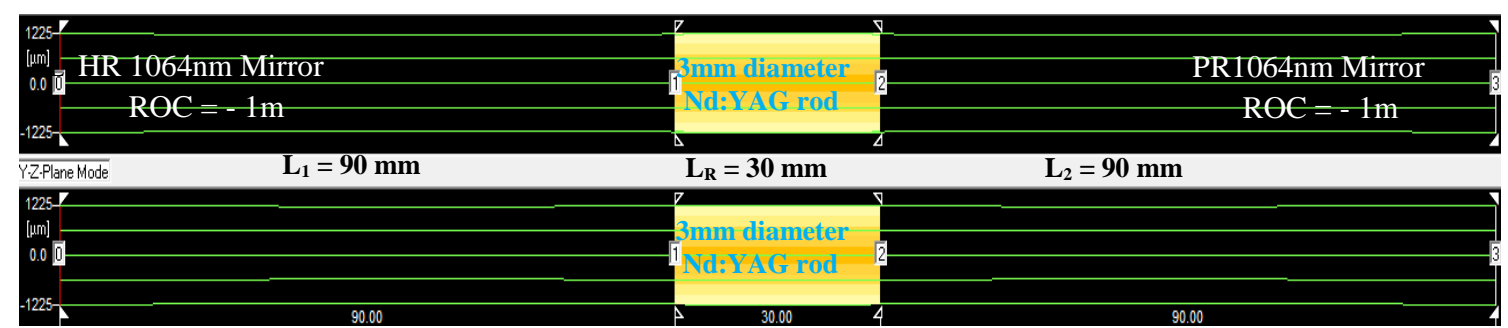

Fig. 5. LASCAD representation of the symmetric laser resonant cavity for the efficient production of multimode solar laser power. $\mathrm{L}_{1}$ and $\mathrm{L}_{2}$ represent the separation length of the high reflection (HR) mirror and partial reflection (PR) mirror, respectively, to the AR end faces of the $3 \mathrm{~mm}$ diameter Nd:YAG rod with length $L_{R}$.

HR1064nm rear mirror and PR1064 nm output mirror of different Radius of Curvature (RoC), PR1064nm output mirror of different reflectivity (R) were tested individually to optimize laser output power. For the $3.0 \mathrm{~mm}$ diameter, $\mathrm{L}_{\mathrm{R}}=30 \mathrm{~mm} \mathrm{Nd}$ :YAG rod, the amount of absorption and scattering losses was $2 \alpha \mathrm{L}_{\mathrm{R}}=2.1 \%$. Assuming 0.4\% imperfect HR1064 nm and AR1064 nm mirror coating losses, the round-trip losses were increased to $2.2 \%$. The diffraction losses depend heavily on rod diameter, resonator length and $\mathrm{RoC}$ of the resonator mirrors, as indicated in Fig.5. For the optimized cavity length of

$\mathrm{L}_{1}=\mathrm{L}_{2}=90 \mathrm{~mm}$ and $\mathrm{RoC}_{1}=\mathrm{RoC}_{2}=-1 \mathrm{~m}, \mathrm{LASCAD}^{\odot}$ beam propagation method gave $0.1 \%$ diffraction loss, resulting finally in a total round-trip loss of $2.3 \%$ for calculating multimode solar laser power in LASCAD analysis. Consequently, $15.6 \mathrm{~W}$ solar laser power was numerically calculated. The laser beam quality $\mathrm{M}_{\mathrm{x}}{ }^{2} \approx \mathrm{M}_{\mathrm{y}}{ }^{2}=16.8$ factors were also numerically obtained. By taking into account the numerically calculated threshold solar power of 390W, solar laser slope efficiency of 5.6\% was numerically obtained.

\section{Continuous-wave 1064nm Nd:YAG solar laser oscillation experiment and laser beam quality measurement}

\subsection{Continuous-wave $1064 \mathrm{~nm} \mathrm{Nd}: Y A G$ solar laser oscillation experiment}

Based on the ZEMAX ${ }^{\odot}$ and LASCAD $^{\odot}$ numerically optimized design parameters of the solar laser system in Section 3, a solar laser prototype, as shown by the inset photos of Fig. 2, was designed, built and finally tested during August, 2018 in Lisbon. The $3.0 \mathrm{~mm}$ diameter, $30 \mathrm{~mm}$ length Nd:YAG singlecrystal rod was AR1064 nm coated ( $\leq 0.2 \% @ 1064 \mathrm{~nm}$ ) on both end faces. To achieve maximum solar laser output power, $\mathrm{a} \mathrm{R}_{1}=99.8 \%$ rear mirror with $\mathrm{RoC}_{1}=-1.0 \mathrm{~m}$ and $\mathrm{R}_{2}=94 \%, \mathrm{RoC}_{2}=-1.0 \mathrm{~m}$ output coupler were mounted $\mathrm{L}_{1}=\mathrm{L}_{2}=90 \mathrm{~mm}$ away from the AR $1064 \mathrm{~nm}$ output faces of the laser rod, as shown in Fig. 5. By varying the rotation angle of the shutter in Fig.1, different input solar power at the focus were measured by a Molectron Power Max 500D power meter and consequently different incoming solar power were calculated based on the reflectance of both heliostat $(93.5 \%)$ and the parabolic mirror $(80.0 \%)$. Output laser power was measured by a Thorlabs PM1100D power meter. Direct solar irradiance was measured simultaneously during lasing with a Kipp \& Zonen pyroheliometer. It varied between $700 \mathrm{~W} / \mathrm{m}^{2}$ and $770 \mathrm{~W} / \mathrm{m}^{2}$ during the experiments. For $700 \mathrm{~W} / \mathrm{m}^{2}$ solar irradiance, $15.3 \mathrm{~W}$ continuous-wave $1064 \mathrm{~nm}$ solar laser power was successfully registered, corresponding to $17.0 \mathrm{~W} / \mathrm{m}^{2}$ collection efficiency at $630 \mathrm{~W}$ incoming solar power level. This value was 1.48 times more than the previous record of $11.7 \mathrm{~W} / \mathrm{m}^{2}$ by the PROMES.CBRS heliostat-parabolic mirror system in 2013 [18]. 


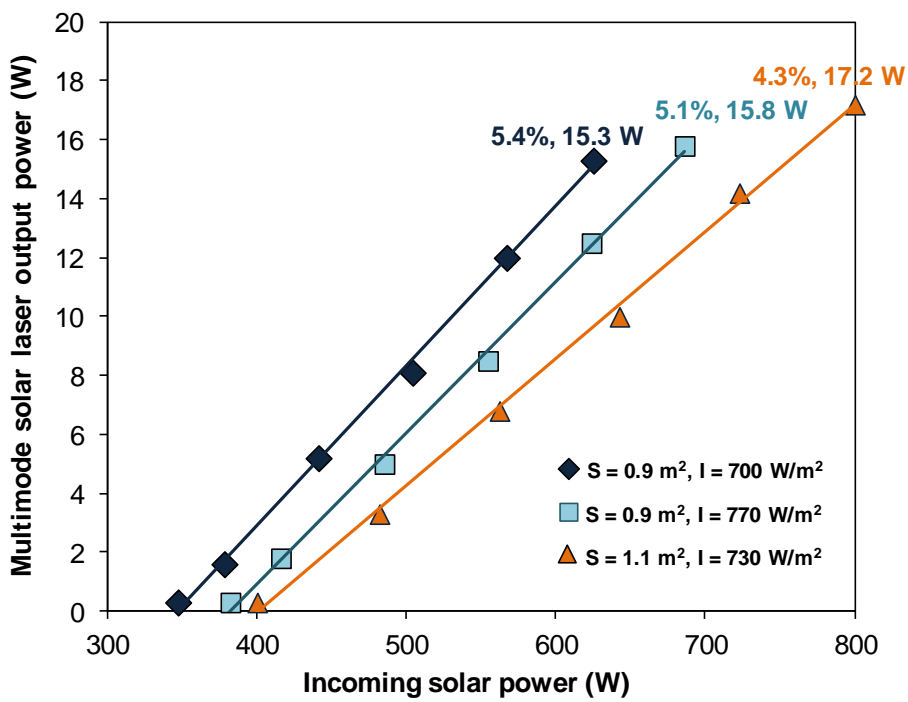

Fig. 6. Solar laser output power versus incoming solar power. The $\mathrm{R}_{1}=99.8 \%$ rear mirror with $\mathrm{RoC}_{1}=-1.0 \mathrm{~m}$ and the $\mathrm{R}=94 \%$ output mirror with $\mathrm{RoC}_{2}=-1.0 \mathrm{~m}$ were mounted $\mathrm{L}_{1}=\mathrm{L}_{2}=90 \mathrm{~mm}$ away from the $\mathrm{AR} 1064 \mathrm{~nm}$ output faces of the laser rod.

For $347 \mathrm{~W}$ threshold incoming solar power, 5.40\% slope efficiency and $2.43 \%$ solar-to-laser power conversion efficency were calculated, being 2.3, 2.15 times respectivelly more than the previous records with side-pumping configuration. Very interestingly, 5.40\% slope efficiency in side-pumping approach was already 1.08 times more than other Nd:YAG solar lasers in end-side-pumped configuration [14] and it was only $19.4 \%$ less than the record-high slope efficiency by $\mathrm{Cr}$ :Nd:YAG solar laser in end-side-pumped configuration [15]. For $770 \mathrm{~W} / \mathrm{m}^{2}$ solar irradiance, $15.8 \mathrm{~W}$ continuous-wave $1064 \mathrm{~nm}$ solar laser power was measured, resulting in $17.6 \mathrm{~W} / \mathrm{m}^{2}$ solar laser collection efficiency, $5.1 \%$ slope efficiency and $2.45 \%$ solar-to-laser conversion efficiency, as shown in Fig. 6. During solar laser oscillation experiment, we also studied carefully the influence of radius of curvature on output solar laser powers. For $770 \mathrm{~W} / \mathrm{m}^{2}$ solar irradiance, $\mathrm{RoC}_{1}=\mathrm{RoC}_{2}=-1.0 \mathrm{~m}$ resulted in the maximum solar laser power of $15.8 \mathrm{~W}$. Other RoCs produced less laser output power. Parabolic mirror with $1.1 \mathrm{~m}^{2}$ effective collection area was also used to test the solar laser output performances. For $730 \mathrm{~W} / \mathrm{m}^{2}$ solar irradiance, $17.2 \mathrm{~W}$ solar laser power was measured. The threshold incoming solar power was about $400 \mathrm{~W}$, leading to $15.6 \mathrm{~W} / \mathrm{m}^{2}$ collection efficiency and $4.3 \%$ laser slope efficiency.

\subsection{Continuous-wave 1064nm solar laser beam quality measurement}

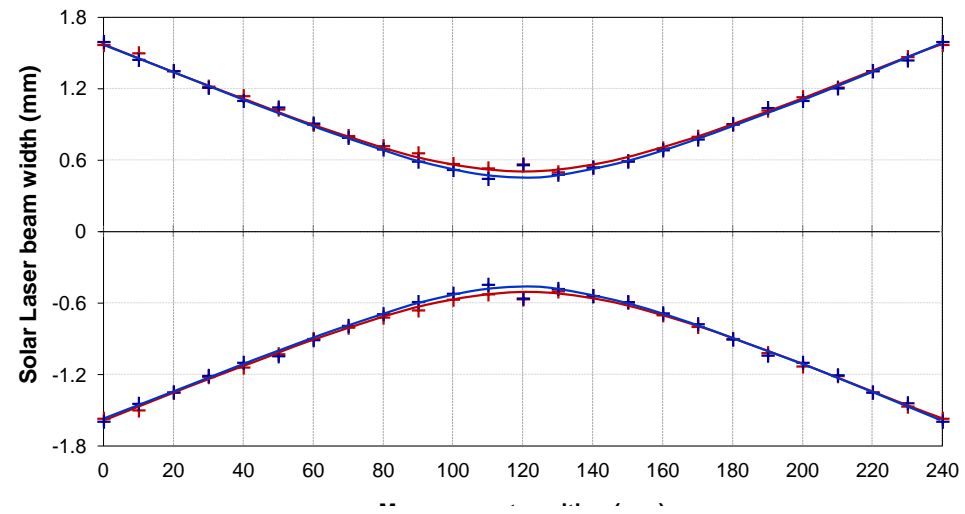

Fig. 7. Caustic fit measurements of the multimode solar laser beam.

The laser beam quality $\mathrm{M}^{2}$ factors were measured according to ISO 11146-1 standards, by using a CINOGY UVNIR beam profiler - CinCam CMOS. The measured solar laser beam profiles along the beam caustic are shown in Fig. 7. The correspondingly solar laser beam widths, along with the extrapolated hyperbolic plot of the measured 
data, are illustrated for measurement positions varying between 0 and $240 \mathrm{~mm}$. For $\operatorname{RoC}_{1}=\operatorname{RoC}_{2}=-1.0 \mathrm{~m}$,

$\mathrm{M}_{\mathrm{x}}{ }^{2} \approx 17.1$ and $\mathrm{My}^{2} \approx 19.0$ were experimentally determined, slightly different than the numerical previsions of $\mathrm{M}_{\mathrm{x}}{ }^{2} \approx \mathrm{M}_{\mathrm{y}}{ }^{2}=16.8$ by LASCAD ${ }^{\odot}$ in Section 3. The discrepancy of $\mathrm{M}^{2}$ in both $\mathrm{X}$ and $\mathrm{Y}$ planes might be caused by solar tracking errors in both azimuth and altitude axis during laser beam profiling process.

\section{The Production of High-Quality Laser Beams with Lower-Order Modes}

Laser emission at lower-order modes, such as in doughnut-shaped profile, enables novel fundamental insights in light-matter interactions and different approaches to modifying materials through laser-based processing $[22,23]$. Therefore, attentions were paid to the efficient design and production of high-quality solar laser beam at lower-order mode by integrating the solar laser head in Fig. 2 into the asymmetric resonator scheme in Fig.5. The use of the $3.0 \mathrm{~mm}$ diameter $30 \mathrm{~mm}$ length Nd:YAG rod within the two-dimensional semi-cylindrical pump cavity and the adoption of the asymmetric resonator configuration, with concave end mirrors of large ROC, were essential for the efficient emission of solar laser beams with low-order-mode profile. The asymmetric resonator configuration has shown to provide a large spatial overlap between the lower-order modes and pump mode volume $[17,19] . \mathrm{L}_{1}$ and $\mathrm{L}_{2}$ represented the separation length of the HR and PR mirrors to their nearest end face of the laser rod, respectively. These parameters together with HR and PR mirrors reflectivity $(\mathrm{R})$ and radius of curvature (RoC) were optimized to achieve the lowest laser beam divergence and, thus, the highest fundamental mode power. $\mathrm{L}_{1}$ was a key parameter for achieving the optimum mode overlap. If $\mathrm{L}_{1}$ increased the fundamental mode size grown up, especially for relatively high input power level. Thus, if we aimed to obtain efficient extraction of lower-mode laser, the laser should operate not far from the optically stable region, where the fundamental mode size was more sensitive to thermal focus fluctuations. $L_{1}$ parameter together with $H R$ and PR mirrors reflectivity $(R)$ and RoC were optimized to achieve the high-quality low-order-mode laser beam with good profiles.

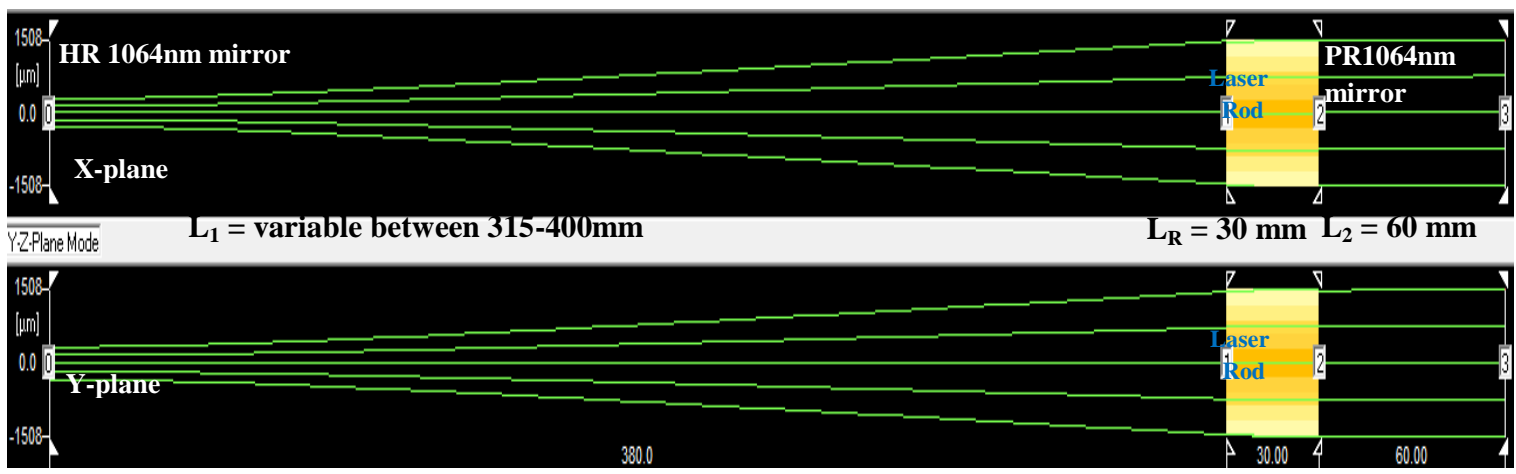

Fig.8 Asymmetric laser resonant cavity was adopted for the efficient extraction of lower-order mode solar laser power. $\mathrm{L}_{1}$ and $\mathrm{L}_{2}$ represent the separation length of the high reflection (HR) mirror and partial reflection (PR) mirror, respectively, to the end face of the laser rod with length $L_{R}=30 \mathrm{~mm}$.

In Fig.8, $\mathrm{RoC}_{1}=\mathrm{RoC}_{2}=-5 \mathrm{~m}$ resonator mirror were adopted in lower-order mode solar laser emission experiment. A CINOGY UV-NIR beam profiler CinCam CMOS was used for monitoring the laser beam profile during the experiments. As shown in Table 1 , when $\mathrm{L}_{2}$ was fixed at $60 \mathrm{~mm}$, then $\mathrm{L}_{1}$ played a very important role in controlling the number of lasing modes and power. For $\mathrm{L}_{1}=315 \mathrm{~mm}$ at $770 \mathrm{~W} / \mathrm{m}^{2}$ solar irradiance, $3.9 \mathrm{~W}-4.0 \mathrm{~W}$ solar laser power with $\mathrm{TEM}_{11}$ mode profiles were observed. When $\mathrm{L}_{1}$ was increased to $370 \mathrm{~mm}$, the $\mathrm{TEM}_{11}$ profile was reduced to a quasi-TEM $0_{00}$-mode profile with $3.5 \mathrm{~W}$ solar laser power. For $\mathrm{L}_{1}=380 \mathrm{~mm}$, we took advantage of the variation of solar irradiance, from $700 \mathrm{~W} / \mathrm{m}^{2}$ to $810 \mathrm{~W} / \mathrm{m}^{2}$, during the day, to achieve different $\mathrm{TEM}_{01}$ mode profile at different laser power level, varying between $2.6 \mathrm{~W}$ and $2.9 \mathrm{~W}$. Due to the power-dependent thermal lensing and mode-matching sensitivity of the laser material, different solar irrandiance led to different thermal lensing effect, and hence the production of $\mathrm{TEM}_{01}{ }^{*}$ beams with different dimensions, power and contrast. For these reasons, strong thermal lensing at $810 \mathrm{~W} / \mathrm{m}^{2}$ irradiance had the tendency to change the $\mathrm{TEM}_{01}{ }^{*}$ doughnut-shaped beam into an aproximate $\mathrm{TEM}_{01}{ }^{*}+\mathrm{TEM}_{00}$ mode beam at $2.7 \mathrm{~W}$ laser power. For $\mathrm{L}_{1}=390 \mathrm{~mm}, \mathrm{TEM}_{01}$ mode laser beam was obtained. We took again the advantage of the variation of solar irradiance during another day, from 
$700 \mathrm{~W} / \mathrm{m}^{2}$ to $840 \mathrm{~W} / \mathrm{m}^{2}$, to achieve different $\mathrm{TEM}_{01}$ mode profiles at different laser power level, varying between $2.5 \mathrm{~W}$ and $3.2 \mathrm{~W}$. For $840 \mathrm{~W} / \mathrm{m}^{2}$ solar irradiance, strong thermal lensing had the tendency to transform the two-mode laser beam also into a $\mathrm{TEM}_{00}$-mode beam profile at $2.5 \mathrm{~W}$ laser power level. Slight angular adjustment of HR1064nm mirror also helped the successful transformation of TEM $\mathrm{T}_{01}$ beam to $\mathrm{TEM}_{00}$ beam [24].

Table 1, Summary of $\mathrm{TEM}_{11}, \mathrm{TEM}_{01^{*}}, \mathrm{TEM}_{10}$ and $\mathrm{TEM}_{00}$ mode laser beam profiles by side-pumping the $3 \mathrm{~mm}$ diameter $30 \mathrm{~mm}$ length rod with the two-dimensional semi-cylindrical pump cavity.

\begin{tabular}{|c|c|c|c|c|}
\hline $\begin{array}{l}\text { TEM }_{11} \\
\text { Mode }\end{array}$ & $\begin{array}{c}4.0 \mathrm{~W}, \mathrm{~L}_{1} 315 \mathrm{~mm} \\
770 \mathrm{~W} / \mathrm{m}^{2}\end{array}$ & $\begin{array}{c}3.9 \mathrm{~W}, \mathrm{~L}_{1} 315 \mathrm{~mm} \\
770 \mathrm{~W} / \mathrm{m}^{2}\end{array}$ & $\begin{array}{c}3.7 \mathrm{~W}, \mathrm{~L}_{1} 360 \mathrm{~mm} \\
770 \mathrm{~W} / \mathrm{m}^{2}\end{array}$ & $\begin{array}{c}3.5 \mathrm{~W}, \mathrm{~L}_{1} 370 \mathrm{~mm} \\
770 \mathrm{~W} / \mathrm{m}^{2}\end{array}$ \\
\hline $\begin{array}{l}\text { Constant } \\
\text { irradiance }\end{array}$ & & & & \\
\hline $\begin{array}{l}\text { TEM }_{01}^{*} \\
\text { Mode }\end{array}$ & $\begin{array}{c}2.9 \mathrm{~W}, \mathrm{~L}_{1} 380 \mathrm{~mm} \\
700 \mathrm{~W} / \mathrm{m}^{2}\end{array}$ & $\begin{array}{c}2.7 \mathrm{~W}, \mathrm{~L}_{1} 380 \mathrm{~mm} \\
720 \mathrm{~W} / \mathrm{m}^{2}\end{array}$ & $\begin{array}{c}2.6 \mathrm{~W}, \mathrm{~L}_{1} 380 \mathrm{~mm} \\
760 \mathrm{~W} / \mathrm{m}^{2}\end{array}$ & $\begin{array}{c}2.7 \mathrm{~W}, \mathrm{~L}_{1} 380 \mathrm{~mm} \\
810 \mathrm{~W} / \mathrm{m}^{2}\end{array}$ \\
\hline $\begin{array}{l}\text { Variable } \\
\text { irradiance }\end{array}$ & & & & \\
\hline $\begin{array}{l}\mathrm{TEM}_{01} \\
\text { Mode }\end{array}$ & $\begin{array}{c}3.2 \mathrm{~W}, \mathrm{~L}_{1} 390 \mathrm{~mm} \\
700 \mathrm{~W} / \mathrm{m}^{2}\end{array}$ & $\begin{array}{c}2.8 \mathrm{~W}, \mathrm{~L}_{1} 390 \mathrm{~mm} \\
760 \mathrm{~W} / \mathrm{m}^{2}\end{array}$ & $\begin{array}{c}2.6 \mathrm{~W}, \mathrm{~L}_{1} 390 \mathrm{~mm} \\
800 \mathrm{~W} / \mathrm{m}^{2}\end{array}$ & $\begin{array}{c}2.5 \mathrm{~W}, \mathrm{~L}_{1} 390 \mathrm{~mm} \\
840 \mathrm{~W} / \mathrm{m}^{2}\end{array}$ \\
\hline $\begin{array}{l}\text { Variable } \\
\text { irradiance }\end{array}$ & & & & \\
\hline $\begin{array}{l}\text { TEM }_{00} \\
\text { Mode }\end{array}$ & $\begin{array}{c}2.8 \mathrm{~W}, \mathrm{~L}_{1} 400 \mathrm{~mm} \\
770 \mathrm{~W} / \mathrm{m}^{2}\end{array}$ & $\begin{array}{c}2.5 \mathrm{~W}, \mathrm{~L}_{1} 400 \mathrm{~mm} \\
790 \mathrm{~W} / \mathrm{m}^{2}\end{array}$ & $\begin{array}{c}2.4 \mathrm{~W}, \mathrm{~L}_{1} 400 \mathrm{~mm} \\
820 \mathrm{~W} / \mathrm{m}^{2}\end{array}$ & $\begin{array}{c}2.3 \mathrm{~W}, \mathrm{~L}_{1} 400 \mathrm{~mm} \\
830 \mathrm{~W} / \mathrm{m}^{2}\end{array}$ \\
\hline $\begin{array}{l}\text { Variable } \\
\text { irradiance }\end{array}$ & & & & \\
\hline
\end{tabular}

For $\mathrm{L}_{1}=400 \mathrm{~mm}$, even with variable solar irradiance between $770 \mathrm{~W} / \mathrm{m}^{2}$ to $830 \mathrm{~W} / \mathrm{m}^{2}, \mathrm{TEM}_{00}$-mode solar laser beam always appeared. $2.8 \mathrm{~W}$ was the highest $\mathrm{TEM}_{00}$-mode solar laser power measured at $770 \mathrm{~W} / \mathrm{m}^{2}$ irradiance, corresponding to $2.5 \mathrm{~W} / \mathrm{m}^{2}$ fundamental mode solar laser collection efficiency. $\mathrm{TEM}_{00}$ mode laser emissions with excellent beam profiles, at $2.5 \mathrm{~W}, 2.4 \mathrm{~W}$, and $2.3 \mathrm{~W}$ power level were respectively measured for the solar irradiance varying between $790 \mathrm{~W} / \mathrm{m}^{2}$ and $830 \mathrm{~W} / \mathrm{m}^{2}$.

\section{Discussions}

During the experiment, we have noticed a very interesting phenomenon that the solar laser slope efficiency actually reached the maximum value of $5.4 \%$ at $700 \mathrm{~W} / \mathrm{m}^{2}$ solar irradiance when the Sun reached us through a homogeneous, thin layer of cloud. Only 5.1\% slope efficiency was measured at $770 \mathrm{~W} / \mathrm{m}^{2}$ irradiance through a more transparent sky condition. Before reaching the laser rod, a considerable part of IR solar radiation from the Sun was already absorbed by the cloud, three conclusions may therefore be drawn. 1: Our solar laser can offer higher slope efficiency with the thin layer of could in the sky than without it. 2: More importantly, for cloudless sunny days, an IR filter may be directly integrated to the solar laser head so that only less than $0.9 \mu \mathrm{m}$ wavelength solar radiation is used to pump the rod. 3: It is hence not difficult to infer that another UV filter may also be added to cut undesirable 
solar radiation below $0.45 \mu \mathrm{m}$. The above mentioned measures may consequently ensure a slope efficiency higher than $5.4 \%$ in future researches.

In solid-state lasers, end-side-pumped scheme usually produces higher laser system efficiency as compared to that by side-pumping approach. Indeed, the end-side-pumped Nd:YAG [13] solar laser enabled 3.1\% solar-to-laser power conversion efficiency, being 1.24 times higher than that of our sidepumped Nd:YAG laser, as given in Fig.6. However, even compared with other Nd:YAG solar laser in end-side-pumping configuration, the measured slope efficiency of 5.4\% is already 1.08 times higher and offered a much better tracking error compensation capacity. It was also only $19.4 \%$ less than that of Cr:Nd:YAG solar laser in end-side-pumped configuration, at $6.7 \%$ slope efficiency and $\mathrm{M}_{\mathrm{x}}{ }^{2} \approx \mathrm{M}_{\mathrm{y}}{ }^{2}=95$ [15]. In terms of solar laser beam brightness figure of merit, - defined as the ratio between laser power and the product of $\mathrm{M}_{\mathrm{x}}{ }^{2}$ and $\mathrm{M}_{\mathrm{y}}{ }^{2}$ [9], the state-of-the-art $\mathrm{Cr}$ :Nd:YAG solar laser in end-side-pumped configuration [15] only presented a dismal value of $32.5 \mathrm{~W} /(95.0 \times 95.0)=0.0036 \mathrm{~W}$, while our sidepumped Nd:YAG solar laser $15.3 \mathrm{~W} /(17.3 \times 19.0)=0.046 \mathrm{~W}$ brightness figure of merit, being 12.8 times brighter than $0.0036 \mathrm{~W}$ of the $\mathrm{Cr}: \mathrm{Nd}: \mathrm{YAG}$ laser.

The most common Nd:YAG single-crystal rod was used in this research, due to its excellent thermal conductivity, high quantum efficiency and tensile strength, making it a good candidate to produce solar laser radiation efficiently. It is also worth noting that this novel scheme is also valid for pumping other solar laser materials such as Cr:Nd:YAG ceramic medium [10], Nd:Ce:YAG [25], alexandrite singlecrystal media [26], composite laser rods such as YAG-Nd:YAG-YAG undoped-doped-undoped, and $\mathrm{Nd}$ :YAG grooved rods [14]. New architecture for solar pumped lasers that uses a luminescent solar concentrator [27], diffusive scattering cooling liquid [28] can also be applied to further increase our solar-to-laser conversion efficiency.

\section{Conclusions}

The side-pumped Nd:YAG solar laser head was composed of the fused silica aspheric lens and the two-dimensional semi-cylindrical pump cavity, within which the $3.0 \mathrm{~mm}$ diameter, $30 \mathrm{~mm}$ length Nd: YAG single-crystal rod was efficiently pumped. Optimum optical pumping system design parameters were found through ZEMAX $^{\odot}$ software. Solar laser power and laser beam parameters were optimized through $\mathrm{LASCAD}^{\odot}$ numerical analysis. $15.3 \mathrm{~W}$ continuous-wave multimode solar laser power was successfully measured, corresponding to $17.0 \mathrm{~W} / \mathrm{m}^{2}$ solar laser collection efficiency, $5.40 \%$ slope efficiency and $2.43 \%$ solar-to-laser power conversion efficency, being 1.45, 2.3, 2.15, respectively, more than the previous records by Nd:YAG rod in side-pumping configuration.

\section{Acknowledgement}

Financial support of the strategic project (UID/FIS/00068/2013) of the Science and Technology Foundation of Portuguese Ministry of Science, Technology and Higher Education (FCT - MCTES) is acknowledged. The fellowship grants PD/BD/128267/2016, PD/BD/142827/2018, SFRH/BPD/125116/2016 of B. D. Tibúrcio, D. Garcia and C. R. Vistas, respectively, are acknowledged.

\section{References}

[1] M. Lando, J. Kagan, Y. Shimony, Y. Kalishy, Y. Noter, A. Yogev, S. Rotman, S. Rosenwaks, Solar-pumped solid state laser program, Proc. SPIE 3110 (1997) 196-201.

[2] T. Yabe, T. Ohkubo, T. Dinh, H. Kuboyama, J. Nakano, K. Okamoto, Demonstration of solar-pumped laserinduced magnesium production from magnesium oxide, in: S. N. Mathaudhu, W. H. Sillekens, N. R. Neelameggham, N. Hort (Eds.), Magnesium Technology 2012, Springer, Cham, 2012, pp.55-58. 
[3] T. Yabe, S. Uchida, K. Ikuta, K. Yoshida, C. Baasandash, M. S. Mohamed, Y. Sakurai, Y. Ogata, M. Tuji, Y. Mori, Y. Satoh, T. Ohkubo, M. Murahara,A. Ikesue, M. Nakatsuka, T. Saiki, S. Motokoshi, C. Yamanaka, Demonstrated fossil-fuel-free energy cycle using magnesium and laser, Appl. Phys. Lett. 89 (2006) 261107261123.

[4] C.G. Young, A sun pumped cw one-watt laser, Appl. Opt. 5 (1966) 993-997.

[5] H. Arashi, Y. Oka, N. Sasahara, A. Kaimai, M. Ishigame, A solar-pumped cw 18 W Nd:YAG laser, Jpn. J. Appl. Phys. 23 (1984) 1051-1053.

[6] M. Weksler, J. Shwartz, Solar-pumped solid-state lasers, IEEE J. Quantum Electron. 24 (1988) 1222-1228.

[7] R.M.J. Benmair, J. Kagan, Y. Kalisky, Y. Noter, M. Oron, Y. Shimony, A. Yogev, Solar-pumped Er, Tm, Ho: YAG laser, Opt. Lett. 15 (1990) 36-38.

[8] D. Jenkins, M. Lando, J. O'Gallagher, R. Winston, A solar-pumped Nd:YAG laser with a record efficiency of 4.7 watt/m2, Bulletin of Israel Physics Society (1996) 101.

[9] M. Lando, J. Kagan, B. Linyekin, V. Dobrusin, A solar pumped Nd:YAG laser in the high collection efficiency regime, Opt. Commun. 222 (2003) 371-381.

[10] T. Yabe, T. Ohkubo, S. Uchida, M. Nakatsuka, T. Funatsu, A. Mabuti, A. Oyama, Y. Nakagawa, T. Oishi, K. Daito, B. Behgol, Y. Nakayama, M. Yoshida, S. Motokoshi, Y. Sato, C. Baasandash, High efficiency and economical solar energy pumped laser with Fresnel lens and chromium co-doped laser medium, Appl. Phys Lett. 90 (2007) 261120-261123.

[11] D. Liang, J. Almeida, Highly efficient solar-pumped Nd:YAG laser, Opt. Express 19 (2011) 26399-26405.

[12] T.H. Dinh, T. Ohkubo, T. Yabe, H. Kuboyama, 120 watt continuous wave solar-pumped laser with a liquid light-guide lens and a Nd:YAG rod, Opt. Lett. 37 (2012) 2670-2672.

[13] D. Liang, J., Almeida, C. R. Vistas, E. Guillot, Solar-pumped Nd:YAG laser with $31.5 \mathrm{~W} / \mathrm{m}^{2}$ multimode and $7.9 \mathrm{~W} / \mathrm{m}^{2} \mathrm{TEM}_{00}$-mode collection efficiencies, Sol. Energ. Mater. Sol. Cells 159 (2017) 435-439.

[14] C. Zhao, Z. Guan, H. Zhang, How to harvest efficient laser from solar light, Proc. SPIE 10511 (2018)

[15] D. Liang, J., Almeida, C. R. Vistas, B. D. Tibúrcio, J. Almeida, Solar-pumped Cr:Nd:YAG ceramic laser with 6.7\% slope efficiency, Sol. Energ. Mater. Sol. Cells 185 (2018) 75-79.

[16] J. Almeida, D. Liang, E. Guillot, Improvement in solar-pumped Nd:YAG laser beam brightness, Opt. Laser Tech. 44 (2012) 2115-2119.

[17] D. Liang and J. Almeida, Solar-pumped TEM $0_{00}$-mode Nd:YAG laser, Opt. Express 21 (2013) 25107-25112.

[18] D. Liang, J. Almeida, E. Guillot, Side-pumped continuous-wave Cr:Nd:YAG ceramic solar laser, Appl. Phys. B - Laser and Optics 111 (2013) 305-311.

[19] C. R. Vistas, D. Liang, J. Almeida and E. Guillot, TEM00 mode Nd:YAG solar laser by side-pumping a grooved rod, Opt. Commun. 366 (2016) 50-56.

[20] Standard tables for reference solar spectral irradiances: direct normal, and hemispherical on $37^{\circ}$ tilted surface, ASTM Standard G173-03 (2012).

[21] Z. Bin, C. Zhao, J. He, S. Yang, The study of active medium for solar-pumped solid-state lasers, Acta Opt. Sin. 27 (2007) 1797-1801.

[22] M. Duocastella, C.B. Arnold, Bessel and annular beams for materials processing, Laser Photon. Rev. 6 (2012) 607-621.

[23] O. Prakash, D.N. Astadjov, P. Kumar, R. Mahakud, J. Kumar, S.V. Nakhe, S.K. Dixit, Effect of spatial coherence on the focusability of annular laser beams, Opt. Commun. 290 (2013) 1-7.

[24] T. Masuda, M. Iyoda, Y. Yasumatsu, M. Endo, Low-concentrated solar-pumped laser via transverse excitation fiber-laser geometry, Opt. Lett. 42 (2017) 3427-3430.

[25] Sh. Payziyev and Kh. Makhmudov, Solar-pumped Nd:YAG laser efficiency enhancement using Cr:LiCAF frequency down- shifter, Opt. Commun. 380 (2016) 57-60.

[26] M. Lando, Y. Shimony, R. M. J. Benmair, D. Abramovich, V. Krupkin, A. Yogev, Visible solar-pumped lasers, Opt. Mat. 13 (1999) 111-115. 
[27] P. D. Reusswig, S. Nechayev, J. M. Scherer, G. W. Huwang, M.G. Bawendi M. A, Baldo, C. Rotschild, A path to practical solar pumped lasers via radiative energy transfer, Sci. Rep. 5 (2015) 14758.

[28] C. J. C. Smyth, S. Mirkhanov, A. H. Quarterman, and K. G. Wilcox, $27.5 \mathrm{~W} / \mathrm{m}^{2}$ collection efficiency solar laser using a diffuse scattering cooling liquid, Appl. Opt. 57, 15 (2018), pp. 4008-4012. 